\title{
Laue transmission diffraction optics for thin film stress calculation
}

\author{
B. L. French ${ }^{\text {a) }}$ and J. C. Bilello \\ Department of Materials Science and Engineering, Center for Nanomaterials Science, \\ University of Michigan, Ann Arbor, Michigan 48109-2136
}

(Received 14 October 2002; accepted 15 April 2003)

\begin{abstract}
White beam Laue transmission diffraction topography has been used to determine the curvature of a crystalline substrate, from which the stress in the overlaying coating is determined. A detailed analytical method has been developed which correlates the introduction of curvature to the substrate in two orthogonal directions with attendant changes in the dimensions of a given topographic reflection. The size of a given reflection is found to depend on the dimensions of the incident beam, the horizontal and vertical incident beam divergences, the orthogonal curvatures of the crystalline substrate, the camera length, and the indices of the selected reflection. The thermal tests of sputtered polycrystalline $\mathrm{Ta}$ and $\mathrm{Cr}$ films on $\mathrm{Si}$ (100) substrates are used to demonstrate the applicability of this phenomenon. Simultaneous observation of film delamination and quantification of associated stresses is shown to be possible. (C) 2003 American Institute of Physics.
\end{abstract}

[DOI: $10.1063 / 1.1580191]$

\section{INTRODUCTION}

The myriad applications of polycrystalline thin metal films, from magnetic devices to protective coatings, have motivated a need for information regarding their mechanical and adhesive behavior as a function of temperature and load conditions. ${ }^{1}$ Recent work has used synchrotron white beam Laue transmission diffraction topography/radiography to concurrently image the adhesive failure of a polycrystalline Ta film and the structure of its underlying crystalline $\mathrm{Si}$ (100) substrate during thermal testing. ${ }^{2}$ Since $\mathrm{x}$ rays contributing to the topographic image must also pass through the thin film, a radiographic image of the film is superimposed on the topographic image of the substrate, both of which are captured in real time by a $20 \mu \mathrm{m}$ resolution charge coupled device (CCD) camera. The stress evolution of thin films in these experiments has typically been ascertained ex situ using an ancillary stress determination method, and subsequently correlated with the radiographic/topographic images. ${ }^{3}$ In the present work it is shown that the x-ray optics of the aforementioned Laue experiment permit the substrate curvature, and therefore the thin film stress, to be calculated from variations in the dimensions of a given reflection.

In the past, crystal disorientation has been assessed using a transmitted white beam with a horizontal divergence of $1^{\circ}-3^{\circ}$ for the purpose of studying polygonization in metals. ${ }^{4,5}$ Bent-crystal Laue optics have since been implemented as focusing monochromators for high-energy synchrotron $\mathrm{x}$ rays and neutrons. ${ }^{6,7}$ Additionally, there is continued interest in the use of bent-crystal Bragg optics as reflective $\mathrm{x}$-ray lenses. ${ }^{8}$ The current study employs a lowdivergence $(0.6 \mathrm{mrad})$ white beam in transmission, having a cross section large enough to yield substantial radiographic/ topographic information. Thus, the morphology of thin film

\footnotetext{
a) Author to whom correspondence should be addressed; electronic mail: blfrench@engin.umich.edu
}

delamination and concomitant stress can be obtained simultaneously using the substrate itself as a veritable noncontact strain gauge.

\section{EXPERIMENT}

Thin $\mathrm{Ta}$ and $\mathrm{Cr}$ films were grown on the native oxide of $\mathrm{Si}(100)$ wafers by de magnetron sputtering in a vacuum chamber with a base pressure less than $10^{-7}$ Torr. Typical substrates were $1 \mathrm{~cm} \times 4 \mathrm{~cm}$ rectangles having $\langle 110\rangle$ edges. The films were grown to a nominal thickness of $950 \mathrm{~nm}$ at an ultrahigh purity Ar working gas pressure of 2 mTorr. The residual growth stresses in these films were obtained using double crystal diffraction topography (DCDT), and were found to be $-200 \pm 18$ and $+416 \pm 35 \mathrm{MPa}$ in the $\mathrm{Ta}$ and $\mathrm{Cr}$ films, respectively. ${ }^{9}$

Thermal testing of these films was conducted using an apparatus consisting of a heating device/sample holder and a portable CCD x-ray imaging system, described previously by Zhao et al. ${ }^{10}$ This apparatus is shown schematically in Fig. 1.

The Laue transmission diffraction topography experiments were performed on beamline 2-2 at the Stanford Synchrotron Radiation Laboratory. This is a white x-ray beamline having an energy range of $2-40 \mathrm{keV}$ and a divergence of $0.6 \mathrm{mrad}$ for the spot size used. Before thermal testing the CCD camera was placed over the desired Laue reflection; in this case a (133) reflection was selected for superior feature contrast and placement within the confines of the experimental apparatus. Ta and $\mathrm{Cr}$ thin film specimens were heated in air at a rate of $\sim 100{ }^{\circ} \mathrm{C} / \mathrm{min}$ to $585{ }^{\circ} \mathrm{C}$ and held there for 60 min, at which time the heating lamps were turned off and the samples were allowed to cool in air. Additionally, 2 mTorr Ta specimens were thermal tested for shorter durations of 5,8 , 22 , and $43 \mathrm{~min}$ using the same heating rate and holding temperature. Synchrotron white beam Laue transmission diffraction topography/radiography was performed during each thermal test with real-time footage captured on video and high resolution still images captured with a frame-grabbing 


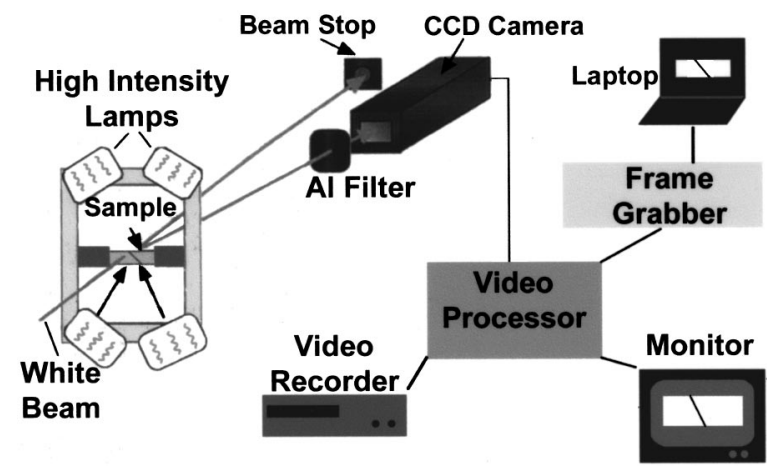

FIG. 1. Schematic of the thermal testing apparatus and $\mathrm{x}$-ray image acquisition system.

computer. The camera length was $33 \mathrm{~cm}$ and the incident beam had a cross section that measured $12.0 \mathrm{~mm} \times 5.2 \mathrm{~mm}$. The resolution of the CCD camera was $20 \mu \mathrm{m} /$ pixel and its aperture diagonal was $18 \mathrm{~mm}$ with an aspect ratio of $3: 4$.

The size of this CCD camera aperture only permitted the observation of one reflection at a time with this experimental configuration. To study the diffraction optics of multiple Laue reflections, three Ta-coated $\mathrm{Si}(100)$ wafers were selected for static transmission Laue topography. The radii of curvature of these wafers were previously determined to be $-15.89 \pm 0.38,238.7 \pm 8.6$, and $14.02 \pm 0.68 \mathrm{~m}$ using DCDT; the corresponding curvatures are simply equal to the inverses of the radii of curvature. Kodak ${ }^{\mathrm{TM}}$ type SR-1 film was placed normal to the beam $10 \mathrm{~cm}$ behind each sample and exposed for $23 \mathrm{~s}$ to record transmission Laue patterns. The incident beam had a cross section that measured $21.0 \mathrm{~mm} \times 7.3 \mathrm{~mm}$ for these exposures, and was normally incident upon the wafer surface. A $1 \mathrm{~mm}$ thick $\mathrm{Al}$ filter was used to reduce the diffracted beam intensity. The negatives developed of each Laue pattern were scanned into computer files so that each reflection could be subsequently examined with imageprocessing software.

\section{DATA ANALYSIS}

Figure 2 is a sequence of topographic/radiographic images depicting the (133) reflection at different stages during the $60 \mathrm{~min}$ thermal test of a $2 \mathrm{mTorr}$ Ta film. The image is featureless initially, but at later times blistering (2109 s) and spalling (2260 s) are evident as the film delaminates from the substrate under high compressive stress. These images can be analyzed to yield information about the delamination kinetics and about the propagation of substrate defects, but that analysis is beyond the scope of this article. In addition to the features in the film and substrate that can be seen in Fig. 2, it is also clear that the actual dimensions of the reflection change during thermal testing. Bending of the substrate causes this focusing effect, as the film becomes strained by thermal expansion mismatch and oxide formation. ${ }^{11}$ Figure 3 is a plot of the changes in the width and height of the reflection as a function of time during the thermal test. The width of the reflection is observed to change drastically, while the

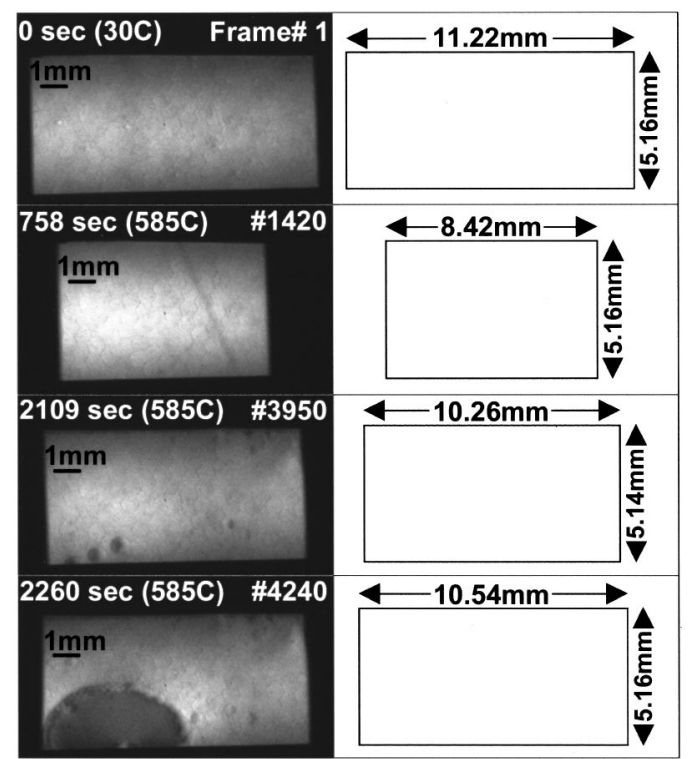

FIG. 2. Sequence of topographic/radiographic images obtained during the $60 \mathrm{~min}$ thermal test of a $2 \mathrm{mTorr}$ Ta thin film. The time from the start of heating is given, as is the temperature. The image is initially featureless; however, at later times blistering is observed (2109 s). Eventually, spalling of the film is evident in the bottom left of the image at $2260 \mathrm{~s}$. At right are schematic drawings of the reflection at each time, with the width and height labeled.

height remains nearly constant. Following is a derivation of equations relating the image size to the instantaneous stress in the thin film.

If a crystalline substrate is introduced to the aforementioned transmission Laue experiment, diffraction conditions are satisfied for all permitted planes by the incident white beam and any reflection may be selected with the CCD camera. With no curvature in the substrate, the plane $(h k l)$ creates the diffraction geometry seen in Fig. 4. In this figure, $\quad \overline{M P}\|\overline{N Q}, \quad \overline{P Q}\| \overline{M N}, \quad \overline{P M} \perp \overline{M N} \perp \overline{M O R}$, and $\overline{R S} \perp \overline{S T} \perp \overline{M O R}$. The substrate surface is perpendicular to the incident beam, and the point of incidence of the beam on plane $(h k l)$ is labeled $O$. The angle of incidence is $\theta$

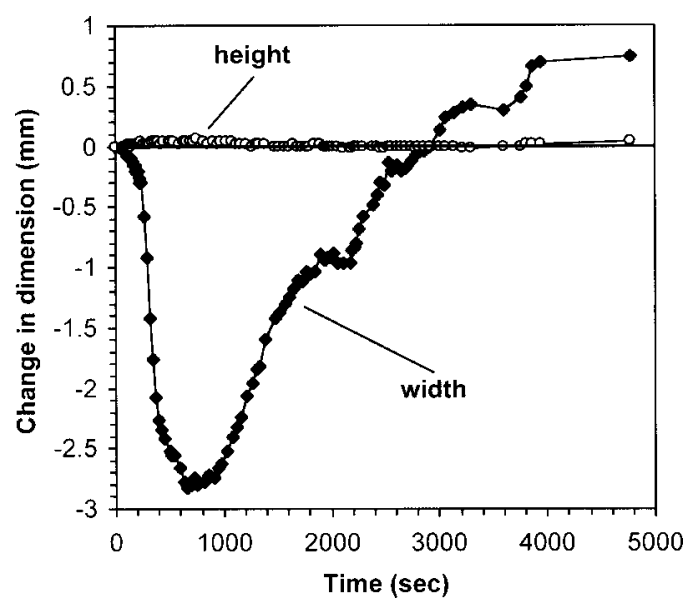

FIG. 3. Plot of the dimension change of the (133) reflection vs time during the $60 \mathrm{~min}$ thermal test of a $2 \mathrm{mTorr}$ Ta thin film. These data are typical of the 2 mTorr Ta films subjected to the $60 \mathrm{~min}$ thermal test described in this study. 


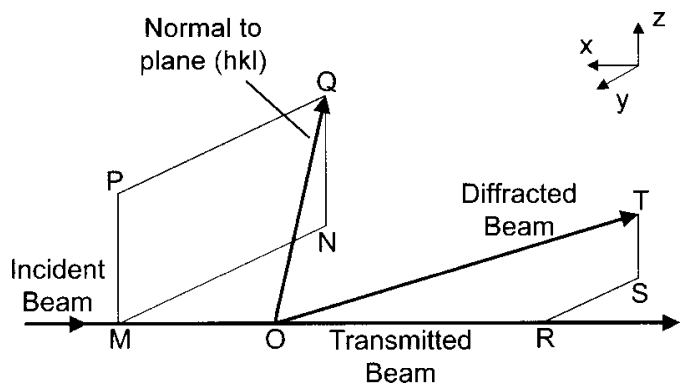

FIG. 4. Diffraction geometry of plane $(h k l)$. The direct beam $\overline{M O R}$ is incident on plane $(h k l)$ at point $O$, yielding the diffracted beam $\overline{O T}$, which intersects the camera plane at point $T$. Line segment $\overline{O Q}$ represents the normal to plane $(h k l)$.

$=\angle M Q O$, and the other angles $2 \theta=\angle R O T, \alpha=\angle M O N$, $\beta=\angle M O P$, and $\delta=\angle N M Q=\angle S R T$, are defined. Points $R, S$, and $T$ lay in the camera plane, where $D=\overline{R S}$ and $H$ $=\overline{S T}$ are coordinates indicating the point of intersection of the diffracted beam and the camera plane. The distance between the substrate and the camera plane is $L=\overline{O R}$.

The line segment $\overline{O Q}$ is normal to diffracting plane $(h k l)$ and has magnitude $\sqrt{h^{2}+k^{2}+l^{2}}$. For a cubic substrate with unit cell edges parallel to the coordinate axes, and a diffracting plane $(h k l), \overline{M O}=h, \overline{M N}=k$, and $\overline{N Q}=l$. It should be emphasized that the plane "indices" $h, k$, and $l$ are only used to specify the angle between the diffracting plane normal and the incident beam. The substrate material need not be cubic; any crystal structure with any orientation could be represented by the schematic of Fig. 4, provided equivalent values of $h, k$, and $l$ are substituted to preserve the correct geometric relationship. In the present work, the $\mathrm{Si}(100)$ substrates were positioned such that the (100) surface was normal to the incident beam and $\langle 110\rangle$ edges were parallel to the $y$ and $z$ axes. Thus, the (133) reflection selected by the CCD camera was represented with "indices" $h=1, k$ $=4.243$, and $l=0$.

If this substrate is bonded to a film in a state of biaxial stress and the substrate is sufficiently thin, this stress will cause curvature of the substrate in two orthogonal planes, as seen in Fig. 5. Assuming this substrate and film assmebly is illuminated by a rectangular beam of white radiation, having dimensions $v_{i}$ along the $y$ axis and $w_{i}$ along the $z$ axis, the beam will experience deflection of the substrate in the $z$ plane as seen in Fig. 6. Figure 6 shows that at the edges of the incident beam the substrate is deflected by an angle $\gamma_{1}$, where

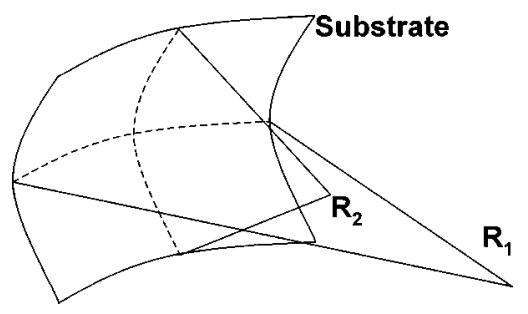

FIG. 5. Crystalline substrate with radii of curvature $R_{1}$ and $R_{2}$.

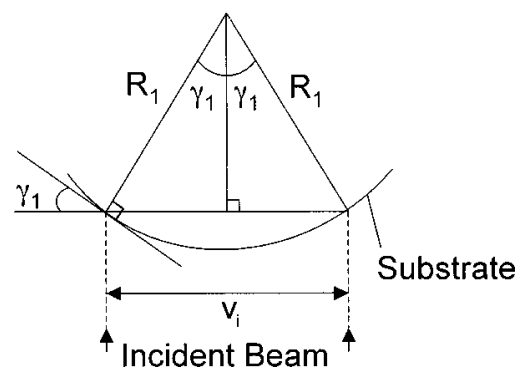

FIG. 6. Plan view of the rectangular white beam incident on a substrate with radius of curvature $R_{1}$. The deflection of the substrate experienced at each edge of the incident beam is $\gamma_{1}$.

$$
\gamma_{1}=\arcsin \left(\frac{\nu_{i}}{2 R_{1}}\right)
$$

Similarly, in the $y$ plane the substrate is deflected by an angle $\gamma_{2}$, where

$$
\gamma_{2}=\arcsin \left(\frac{w_{i}}{2 R_{2}}\right)
$$

Plane ( $h k l)$, described in Fig. 4, will be subject to some change in orientation with respect to the incident beam due to substrate deflection angles $\gamma_{1}, \gamma_{2}$. Initially, assuming the incident beam has zero divergence, the following relationships can be written:

$$
\begin{aligned}
\tan \theta & =\frac{h}{\sqrt{k^{2}+l^{2}}}, \\
\tan \alpha & =k / h, \\
\tan \beta & =l / h, \\
\tan \delta & =l / k .
\end{aligned}
$$

From these relationships it is clear that

$$
\begin{aligned}
& \tan 2 \theta=\frac{2 \sqrt{\tan ^{2} \alpha+\tan ^{2} \beta}}{\tan ^{2} \alpha+\tan ^{2} \beta-1}, \\
& \cos \delta=\frac{\tan \alpha}{\sqrt{\tan ^{2} \alpha+\tan ^{2} \beta}}, \\
& \sin \delta=\frac{\tan \beta}{\sqrt{\tan ^{2} \alpha+\tan ^{2} \beta}} .
\end{aligned}
$$

It is now desirable to relate Eqs. (2) to the dimensions of the diffracted beam in the camera plane. To simplify these relations, it is clear by inspection that only four rays, defining the corners of the rectangular incident beam, need to be considered. The deflection experienced by these four rays due to $\gamma_{1}$ and $\gamma_{2}$ is determined by the convention shown in Fig. 7.

Mapping of these four rays to the camera plane will use the convention shown in Fig. 8. Also labeled in this figure are the divergences possessed by each edge of the incident beam. The rays diffracted to points $r, s, t$, and $u$ in the camera plane will have coordinates $(D, H)$ in that plane determined by the respective deflections experienced by each ray. It 


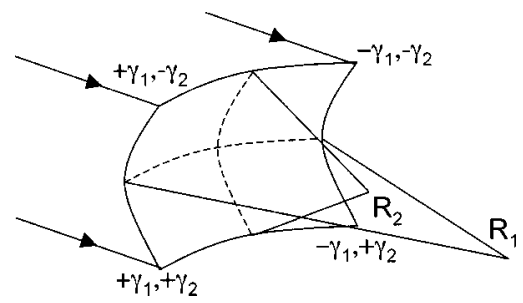

FIG. 7. Illustration of the substrate deflections, $\pm \gamma_{1}, \pm \gamma_{2}$, experienced by the four rays that define the incident beam.

should be noted that these are coordinates relative to each incident ray, not on an absolute coordinate system. From Fig. 4 the following relationship can be written:

$$
\sqrt{H^{2}+D^{2}}=L \tan 2 \theta \text {. }
$$

From Fig. 4 and using Eq. (4),

$$
\begin{aligned}
& D=\cos \delta \sqrt{H^{2}+D^{2}}=L \cos \delta \tan 2 \theta, \\
& H=\sin \delta \sqrt{H^{2}+D^{2}}=L \sin \delta \tan 2 \theta .
\end{aligned}
$$

By substituting Eqs. (3), $D$ and $H$ can be expressed initially as

$$
\begin{aligned}
& D=\frac{2 L \tan \alpha}{\tan ^{2} \alpha+\tan ^{2} \beta-1}, \\
& H=\frac{2 L \tan \beta}{\tan ^{2} \alpha+\tan ^{2} \beta-1} .
\end{aligned}
$$

Now the divergence of the incident rays must be considered. The horizontal and vertical divergences of an unfocused synchrotron white beam are defined as the beam dimensions at the sample divided by the source distance (e.g., a $1 \mathrm{~cm}$ wide beam incident on a sample $20 \mathrm{~m}$ from its source would have a horizontal divergence of $0.5 \mathrm{mrad}$ ).

After the deflections $\gamma_{1}, \gamma_{2}$ and incorporating the divergences, Eqs. (6) become

$$
\begin{aligned}
& D=\frac{2 L \tan \left(\alpha+\gamma_{1}+\phi / 2\right)}{\tan ^{2}\left(\alpha+\gamma_{1}+\phi / 2\right)+\tan ^{2}\left(\beta+\gamma_{2}+\omega / 2\right)-1}, \\
& H=\frac{2 L \tan \left(\beta+\gamma_{2}+\omega / 2\right)}{\tan ^{2}\left(\alpha+\gamma_{1}+\phi / 2\right)+\tan ^{2}\left(\beta+\gamma_{2}+\omega / 2\right)-1},
\end{aligned}
$$

where $\phi$ and $\omega$ are the horizontal and vertical beam divergences, respectively, as labeled in Fig. 8. It is clear from

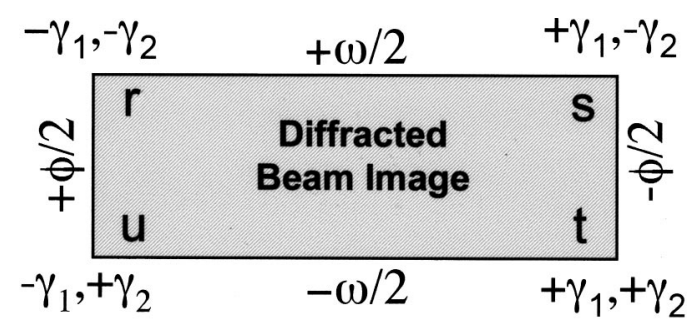

FIG. 8. The four rays representing the corners of the incident beam diffract to points $r, s, t$, and $u$ on the camera plane, and each has experienced the deflection of the substrate $\pm \gamma_{1}, \pm \gamma_{2}$ labeled at that point. The contributions of the horizontal and vertical divergences $\phi$ and $\omega$ to the angles $\alpha$ and $\beta$, respectively, are labeled on the edges along which they apply. these equations that $\gamma_{1}$ and $\gamma_{2}$ effectively rotate the diffracting plane normal about the $z$ and $y$ axes, respectively.

Corners of the diffracted beam illustrated in Fig. 8 can now be assigned coordinates $\left(D_{r}, H_{r}\right),\left(D_{s}, H_{s}\right),\left(D_{t}, H_{t}\right)$, $\left(D_{u}, H_{u}\right)$ in the camera plane. The length of each edge of the reflection can be calculated with these coordinates using the general equation

$$
v_{m n}=v_{m n i}+\left(D_{m}-D_{n}\right)
$$

where $v_{m n}$ is the length of edge $m n$ of the reflection, and $v_{m n i}$ is the length of the corresponding edge of the incident beam. Equivalent expressions can be written for any edge $m n$ of the reflection. Initially, assuming constant beam divergences and a constant substrate curvature, any reflection edge length can be expressed as

$$
v_{m n 0}=v_{m n i}+\Delta v_{m n 0}
$$

Here $v_{m n 0}$ is the initial reflection edge length, and $\Delta v_{m n 0}$ is the difference $\left(v_{m n 0}-v_{m n i}\right)$ due to initial substrate curvature, beam divergence, and experimental geometry. The subsequent change in the reflection size due to a change in substrate curvature can be found by subtracting Eq. (9) from Eq. (8):

$$
\Delta v_{m n}=\left(D_{m}-D_{n}\right)-\Delta v_{m n 0}
$$

$\Delta v_{m n}$ and $\Delta v_{m n 0}$ are measurable experimentally, so Eq. (10) must be solved in terms of substrate deflections $\gamma_{1}$ and $\gamma_{2}$, from which the radii of curvature of the substrate are ultimately obtained. For $\Delta v_{r s}$, substitution of Eq. (7a) for $D_{r}$ and $D_{s}$ yields

$$
\begin{aligned}
\Delta v_{r s}= & \frac{2 L \tan \left(\alpha-\gamma_{1}+\phi / 2\right)}{\tan ^{2}\left(\alpha-\gamma_{1}+\phi / 2\right)+\tan ^{2}\left(\beta-\gamma_{2}+\omega / 2\right)-1} \\
& -\frac{2 L \tan \left(\alpha+\gamma_{1}-\phi / 2\right)}{\tan ^{2}\left(\alpha+\gamma_{1}-\phi / 2\right)+\tan ^{2}\left(\beta-\gamma_{2}+\omega / 2\right)-1} \\
& -\Delta v_{r s 0} .
\end{aligned}
$$

Note that the terms $\gamma_{1}, \gamma_{2}, \phi$, and $\omega$ possess signs according to the convention outlined in Fig. 8. Equation (11) can be solved graphically to yield values of $\gamma_{1}$ and $\gamma_{2}$ given any reflection $(h k l)$, camera length $L$, beam divergences $\phi$ and $\omega$, and change in reflection width $\Delta v_{r s}$. Equivalent expressions can be written for $\left(D_{u}-D_{t}\right),\left(H_{t}-H_{s}\right)$, and $\left(H_{u}\right.$ $-H_{r}$ ) by following the same procedure. In the current study the (133) reflection used could be treated as an ( $h k 0)$ type, as mentioned earlier, such that $\beta=0$. Thus, Eq. (11) could be simplified to

$$
C \cos \left[4 \gamma_{1}-2 \phi\right]+C \cos 4 \alpha-\sin \left[4 \gamma_{1}-2 \phi\right] \approx 0,
$$

where $C=\left(\Delta v_{r s}+\Delta v_{r s 0}\right) / 2 L$. This expression can be solved algebraically, eliminating the need for graphical analysis, such that

$$
\gamma_{1}=\frac{1}{4}\left(\arcsin \left(\frac{C \cos 4 \alpha}{\sqrt{C^{2}+1}}\right)+\arctan C+2 \phi\right) .
$$




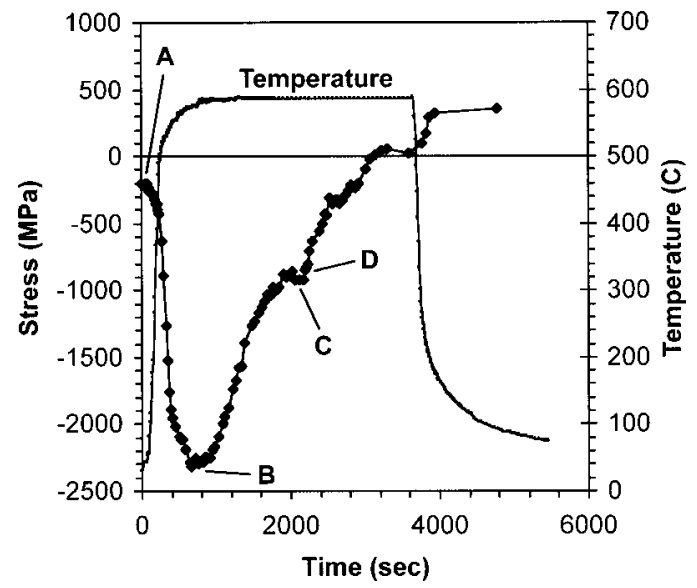

FIG. 9. Stress vs time data from the $60 \mathrm{~min}$ thermal test of a $2 \mathrm{mTorr}$ Ta film, generated from the data plotted in Fig. 3. The temperature profile for thermal testing is plotted on a secondary $y$ axis. The data points labeled A-D were generated from the four topographs shown in Fig. 2, such that point A represents $0 \mathrm{~s}$. Points B, C, and D represent 758, 2109, and $2260 \mathrm{~s}$, respectively.

The fact that $\beta=0$ for this reflection also explains the absence of change in the height of the reflection, as seen in Figs. 2 and 3.

Once $\gamma_{1}$ and $\gamma_{2}$ are known, $R_{1}$ and $R_{2}$ can be calculated from Eqs. (1). The strain in the substrate due to the radius of curvature, $R$, can be expressed as

$$
\varepsilon=\frac{y}{R},
$$

where $\varepsilon$ is the strain and $y$ is the distance from the neutral axis of bending. All that remains is to determine the stresses in the film corresponding to radii of curvature $R_{1}$ and $R_{2}$, using the modified Stoney equation

$$
\sigma=\frac{E_{s}\left(h_{s}\right)^{2}}{6 h_{f}\left(1-v_{s}\right)}\left(\frac{1}{R}-\frac{1}{R_{0}}\right),
$$

where $\sigma$ is the film stress, $E_{s}$ is the substrate elastic modulus, $v_{s}$ is Poisson's ratio of the substrate, $R_{0}$ is the initial radius of curvature, and $h_{s}$ and $h_{f}$ are the thickness of the substrate and film, respectively. ${ }^{12,13}$ It is clear upon inspection that the instantaneous stress is calculated relative to the initial stress in the film, so it is imperative that the initial stress be calculated by some other means to offset the calculated stress data. In this case, double crystal diffraction topography was used. $^{7}$

\section{RESULTS}

The preceding analysis can now be applied to the images obtained from the $60 \mathrm{~min}$ thermal test of the Ta film. Using $E_{s} /\left(1-v_{s}\right)=180.5 \mathrm{GPa}, h_{s}=490 \mathrm{~mm}$, and other constants defined earlier, Fig. 9 is generated from the width data plotted in Fig. 3. Using this plot, events in the stress evolution of this sample can be unambiguously identified and ascribed to specific mechanisms (e.g., blistering or spalling in the film). Subsequent to heating, a large compressive stress is induced. $\mathrm{X}$-ray diffraction performed ex situ reveals that this Ta film has made a complete conversion to base-centered orthorhom-

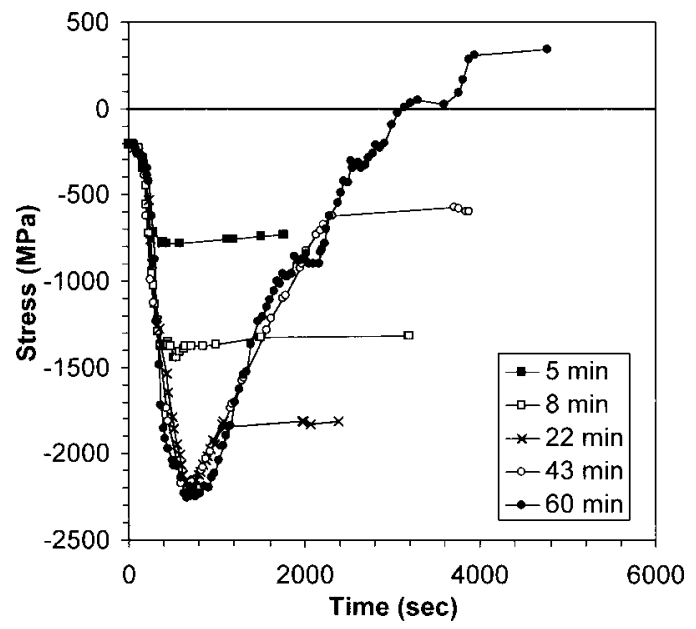

FIG. 10. Stress vs time data from $5,8,22,43$, and $60 \mathrm{~min}$ thermal tests of $2 \mathrm{mTorr}$ Ta films. These samples were heated along the same temperature profile shown in Fig. 9.

bic $\mathrm{Ta}_{2} \mathrm{O}_{5}$, which has approximately half the density of the as-deposited $\beta$-Ta phase. Formation of this less-dense oxide explains the magnitude of compressive stress, which is more than three times larger than that predicted by coefficient of thermal expansion (CTE) mismatch alone. ${ }^{11}$ At a time of about $760 \mathrm{~s}$ a maximum stress is achieved, and a stress relaxation begins. This relaxation is accompanied by the first observation in the radiographic images of thin film blistering, presumably the mechanism responsible for this initial stress relaxation. At about $2000 \mathrm{~s}$, there is a small plateau in the stress versus time data. Radiographic images show that at this point spalling of the film is first evident, and apparently accounts for all further stress relaxation of the film until cooling is initiated. The slope of the curve tends toward zero just before cooling begins, as the stress approaches zero and the driving force for film spallation disappears.

Applying the same methodology used to generate the data plotted in Fig. 9, the 2 mTorr Ta films' stress response to shorter thermal tests was investigated. Stress versus time data from $5,8,22,43$, and 60 min thermal tests is plotted in Fig. 10. Each data series overlaps very closely, which demonstrates the uniformity of both the thermal testing procedure and the thin film response. This uniformity is further illustrated by the fact that the maximum compressive stresses achieved in the 22, 43, and 60 min thermal tests are nearly equal. Another important result of this plot is that for each thermal test duration, most of the stress present in the film just before cooling is retained except for a slight relaxation that can be attributed to thermal stress relief. This stress retention is most likely the result of oxidation-induced stress, and is the subject of future study. A similar phenomenon was observed by Cabral et al. during vacuum anneals of Ta coatings. ${ }^{11}$

Data obtained from the 60 min thermal test of the 2 mTorr $\mathrm{Cr}$ coating yielded the results plotted in Fig. 11. As with the Ta films, stresses much larger than CTE mismatch stress are achieved in the film as the sample is heated to its holding temperature. Again, this can be attributed to oxide formation, as evidenced by ex situ x-ray diffraction scans. At 


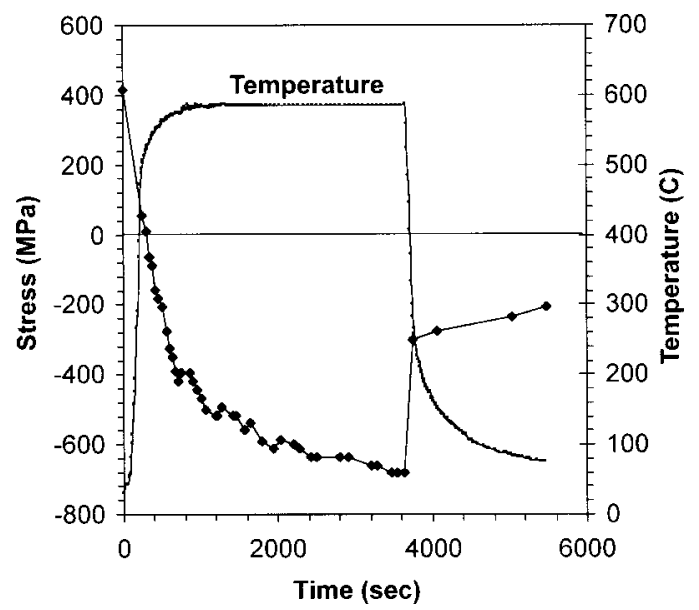

FIG. 11. Stress vs time data from the $60 \mathrm{~min}$ thermal test of a $2 \mathrm{mTorr} \mathrm{Cr}$ film. The temperature profile for thermal testing is plotted on a secondary $y$ axis.

a time of about $700 \mathrm{~s}$ there is a slight reduction in the slope of the stress data, which eventually approaches zero just before cooling is initiated. The complete conversion of the coating into a stable high-temperature oxide could explain this reduction in slope. Another possibility is that an outer oxide scale has formed which becomes increasingly resistant to further oxide penetration, thereby reducing the rate at which compressive stress forms in the film. While this behavior is the subject of further investigation, it is clear that there is no catastrophic stress relaxation as exhibited by the Ta coatings. This is corroborated by the radiographic images of this sample, which showed no evidence of blistering or spalling. Upon cooling the thermal stress in the film is relieved while a compressive stress is retained, presumably due again to the presence of less-dense oxide phases.

As a preliminary check of this technique's validity, it is of interest to compare the slope of the initial stress versus temperature data to predictions based upon the CTE mismatch between the film and the substrate calculated from bulk CTE values. The experimental data from the $\mathrm{Ta}$ and $\mathrm{Cr}$ films yield slopes of 1.3 and $0.9 \mathrm{MPa} /{ }^{\circ} \mathrm{C}$, respectively. The predicted values for the $\mathrm{Ta}$ and $\mathrm{Cr}$ films are $1.1 \mathrm{MPa} /{ }^{\circ} \mathrm{C}$ (15.4\% difference) and $0.8 \mathrm{MPa} /{ }^{\circ} \mathrm{C}(12.5 \%$ difference), respectively. There are two possible explanations for this difference. First, the predicted slopes were calculated using bulk CTE values that might not necessarily describe the behavior of thin films. ${ }^{14}$ Second, while stress due to oxidation of the films should be minimized in this initial stage of the thermal test, some oxidation inevitably occurs which might increase the stress over values predicted solely by thermal expansion mismatch. ${ }^{9}$ This second possibility is supported by the fact that for each film tested, the experimentally determined slope was greater than the predicted slope, as would be the case if there was an added stress component due to oxidation.

All dynamic experimental data presented thus far have been generated from observing the $\mathrm{Si}$ (133) substrate reflection. Since observation of additional reflections is necessary to corroborate this analytical method, static transmission Laue topographs were taken from the three Ta-coated

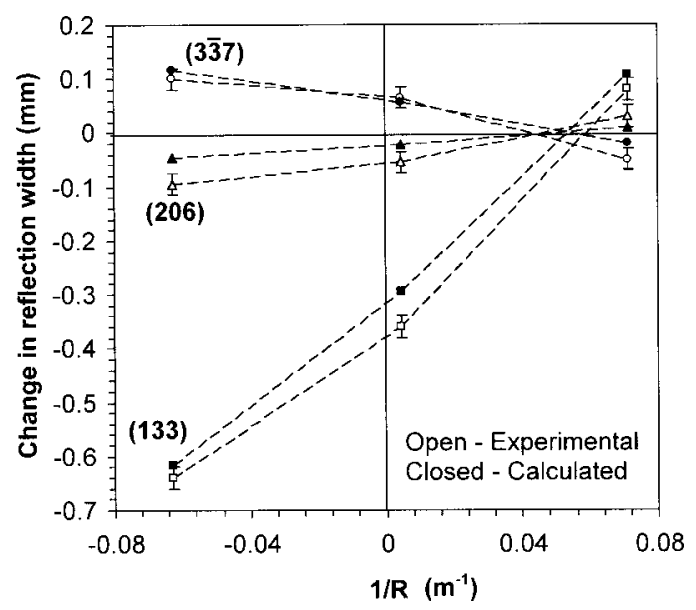

FIG. 12. Calculated change in reflection width (closed symbols) and experimentally measured change in reflection width (open symbols) for three predetermined Si (100) wafer curvatures. The Si (133), (206), and (337) reflections are shown because of their disparate changes in reflection width as a function of wafer curvature. The lines are drawn to guide the eye.

Si (100) wafers of predetermined curvature. The dimensions of many reflections were measured and compared to the dimensions predicted by Eq. (11) (solving for $\Delta v_{r s 0}$ with $\Delta v_{r s}=0$ ) for the given substrate curvatures. Figure 12 illustrates the calculated and experimental results from the Si (133), (206), and (337) reflections, selected for their disparate trends over the given range of wafer curvatures. In terms of the angles $\alpha$ and $\beta$ (defined by Fig. 4) the (133), (206), and (33) reflections represent the conditions $\alpha>\beta$, $\mathrm{a}=\beta$, and $\beta>\alpha$, respectively. While there is generally good agreement between calculated and experimental data, discrepancies could be due to error in the measured camera length, slight misorientation of the wafers within the reference frame outlined in Fig. 4, or nonuniform wafer curvature.

\section{CONCLUSIONS}

Focusing of transmission Laue reflections due to crystal curvature has been described in detail, and its utility as a nondestructive means for simultaneously observing thin film morphology and computing thin film stress has been demonstrated. The effects of camera length, incident beam dimensions, beam divergence, reflection indices, and substrate curvature were taken into account. The dynamic experiments presented here consider the use of an $(h k 0)$ type reflection, and thus the stress is only accurately calculable in one direction. Monitoring of an $(h k k)$ type reflection would provide sensitivity to the substrate curvature in two directions and possibly provide a useful means for studying anisotropically stressed films. ${ }^{15,16}$ Static transmission Laue topography of Ta-coated Si (100) wafers with previously determined curvatures provided corroborating data for $(h k k)$ type reflections, demonstrating good agreement between predicted and experimental data. Plots of predicted reflection dimensions are potentially useful in selecting a reflection to observe for a given application.

The experimental configuration used could resolve changes in stress of about $15 \mathrm{MPa}$ for the samples tested. This resolution can be improved in a number of ways other 
than simply using a higher-resolution CCD camera. First, reducing the substrate thickness or increasing the film thickness effect an improvement in resolution by focusing or defocusing the diffracted beam more for a given shift in stress. Both of these changes can cause excessive substrate curvature and extreme focusing of the selected reflection, which can lead to a loss of topographic/radiographic information and substrate fracture if the stress is sufficiently large. Second, increasing the camera length $L$ or the initial dimensions of the beam, $v_{i}$ and $w_{i}$, induces an increase in resolution. A third means of improving the resolution is to select a more sensitive reflection for observation. In the experiments described here, for example, the use of a (122) type reflection would yield an 11.3 MPa stress resolution. The second and third suggestions improve the "optical leverage" of the technique by allowing the rays that define the diffracted beam to trace longer paths in the camera plane for a given change in stress. ${ }^{17}$ A larger CCD camera aperture would be needed to accommodate any of these changes in a dynamic experiment, because in each case defocusing of the diffracted beam would be augmented in the camera plane relative to the current experimental configuration.

\section{ACKNOWLEDGMENTS}

This work is sponsored, in part, by the ARO under GRANT Nos. DAAD 19-02-1-0335 and DAAG 55-98-1-
0382. The authors would like to thank the USDOE for support of work performed at the Stanford Synchrotron Radiation Laboratory. They also thank Dr. Zofia Rek for her assistance on Beamline 2-2 at SSRL.

${ }^{1}$ H. Windischmann, CRC Crit. Rev. Solid State Mater. Sci. 17, 547 (1992).

${ }^{2}$ Z. B. Zhao, Z. U. Rek, and J. C. Bilello, Philos. Trans. R. Soc. London, Ser. A 357, 2681 (1999).

${ }^{3}$ Z. B. Zhao, J. Hershberger, A. Chiaramonti, Z. U. Rek, and J. C. Bilello, Mater. Res. Soc. Symp. Proc. 524, 81 (1998).

${ }^{4}$ A. Guinier and J. Tennevin, Acta Crystallogr. 2, 133 (1949).

${ }^{5}$ A. Guinier and J. Tennevin, Progress in Metal Physics (Interscience, New York, 1950), Vol. 2, p. 177

${ }^{6}$ P. Suortti, U. Lienert, and C. Schulze, Nucl. Instrum. Methods Phys. Res. A 338, 27 (1994).

${ }^{7}$ V. V. Kvardakov, V. A. Somenkov, J. W. Lynn, D. F. R. Mildner, and H. Chen, Physica B 241-243, 1210 (1998).

${ }^{8}$ V. V. Aristov, Yu. A. Basov, G. N. Kulipanov, V. F. Pindyurin, A. A. Snigirev, and A. S. Sokolov, Nucl. Instrum. Methods Phys. Res. A 274, 390 (1989).

${ }^{9}$ C. L. Kuo, P. E. Vanier, and J. C. Bilello, J. Appl. Phys. 55, 375 (1984).

${ }^{10}$ Z. B. Zhao, J. Hershberger, Z. U. Rek, and J. C. Bilello, Mater. Res. Soc. Symp. Proc. 502, 163 (1998).

${ }^{11}$ C. Cabral, Jr., L. A. Clevenger, and R. G. Shad, Mater. Res. Soc. Symp. Proc. 308, 57 (1993).

${ }^{12}$ G. G. Stoney, Proc. R. Soc. London, Ser. A 82, 172 (1909).

${ }^{13}$ D. W. Hoffman, in Physics of Thin Films, edited by G. Haas and R. E. Thun (Academic, New York, 1965), Vol. 3, p. 211.

${ }^{14}$ C. V. Thompson and R. Carel, J. Mech. Phys. Solids 44, 657 (1996).

${ }^{15}$ D. W. Hoffman and J. A. Thornton, Thin Solid Films 40, 355 (1977).

${ }^{16}$ R. Gontarz and T. Lucinsk, J. Magn. Magn. Mater. 101, 253 (1991).

${ }^{17}$ B. D. Cullity, Elements of X-ray Diffraction, 2nd ed. (Addison-Wesley, Reading, MA, 1978), p. 292. 